\title{
Ultra-High Vacuum Deposition of Pyrene Molecules on Metal Surfaces
}

\author{
Sebastian Schleicher ${ }^{*}, 1,2$, Bogdana Borca ${ }^{1,3}$, Jeff Rawson ${ }^{2,4}$, Frank Matthes ${ }^{1,2}$, Daniel E. Bürgler ${ }^{1,2}$, Paul \\ Kögerler $^{2,4}$, Claus M. Schneider ${ }^{1,2}$ \\ ${ }^{1}$ Peter Grünberg Institute, Electronic Properties (PGI-6), Forschungszentrum Jülich, Germany \\ ${ }^{2}$ Jülich Aachen Research Alliance, Fundamentals of Future Information Technology (JARA-FIT), Forschungszentrum Jülich, Germany \\ ${ }^{3}$ National Institute of Materials Physics, Bucharest, Romania \\ ${ }^{4}$ Institute of Inorganic Chemistry, RWTH Aachen University, Germany
}

Key words: aromatic molecules, sublimation procedure, molecular adsorption, scanning tunneling microscopy

* Corresponding author: e-mail s.schleicher@fz-juelich.de, Phone: +49 2461 613148, Fax: +49 2461614443

Interfacial properties of organic adsorbates featuring aromatic $\pi$-orbitals on metal surfaces play an important role for organic electronics and spintronics. Pyrene is a flat aromatic molecule with a size between ultimately small benzene and extended graphene segments. The deposition of pyrene molecules onto clean and reactive surfaces with a sub-monolayer coverage under ultra-high vacuum (UHV) conditions is challenging, since pyrene is a solid with a high vapor pressure. Here, we present a sublimation procedure under UHV and image pyrene adlayers on in-situ prepared $\mathrm{Au}(111)$ and $\mathrm{Fe} / \mathrm{W}(110)$ substrates by means of low-temperature scanning tunneling microscopy. For Au(111), the molecule-surface interaction is weak as indicated by the specific herringbone reconstruction of the $\mathrm{Au}(111)$ surface that is visible through the self-assembled pyrene adlayer. Pyrene desorption due to weak intermolecular interaction self-limits the growth to one monolayer (ML). On the more reactive 2 to 4 ML thick Fe films on W(110), the molecular order of the pyrene adlayer sensitively depends on the Fe thickness-dependent dislocation pattern at the substrate surface. Irregular arrangements occur for $1 \mathrm{ML}$ Fe and near substrate dislocations for 2 to 4 ML Fe. Self-assembled ordered arrays form predominantly for 2 ML $\mathrm{Fe}$, where the dislocation pattern leaves sufficiently large unperturbed areas between the dislocation lines.

Copyright line will be provided by the publishe

1 Introduction The nanotechnological research fields on molecular electronics [1,2] and molecular spintronics $[3,4]$ aim at tailoring and utilizing single molecules as smallest stable building blocks for electronic devices in order to increase the integration density and energy efficiency of future electronic circuits. Therefore, it is important to study and to control the interaction of single molecules and of single molecular layers with the metal surfaces of electrodes. A recent approach to molecular spintronics employs the adsorption of intrinsically non-magnetic, flat aromatic molecules on ferromagnetic surfaces. The chemisorption on ferromagnetic transition-metal surfaces is governed by strong hybridization of molecular $\pi$-orbitals with spin-split $d$-orbitals of the substrate. As a consequence, the density of states of the adsorbed molecule becomes spin-imbalanced thereby inducing magnetic properties [5-10]. Concurrently, the magnetic moment, the magnetic anisotropy as well as the exchange coupling of the substrate atoms that are directly bound to the molecule are also modified. Thus, the molecule and its immediate neighbor atoms in the substrate form a so-called hybrid molecular magnet showing for instance magnetic hardening [5], i.e. enhanced coercivity compared to the uncovered ferromagnetic surface [5-8]. Chemisorption-induced properties of hybrid molecular magnets critically depend on the local bonding conditions and adsorption geometry [9] making them susceptible to details of the molecular structure, e.g. substitution of single heteroatoms [10], the size of the molecule [6], and the extent of the $\pi$-system [8, $10]$.

Reliable investigations of these phenomena require the controlled adsorption of molecules in sub-monolayer amounts onto well-defined clean single-crystalline surfaces of (transition-) metal surfaces. This usually is only possible under ultra-high vacuum (UHV) conditions. The choice of an appropriate deposition method depends on 
the molecular properties, e.g. size and stability. Small molecules like benzene that are liquid or gaseous at room temperature (RT) and atmospheric pressure can be dosed from the gas phase by injecting small amounts of vapor into the vacuum system [11]. Slightly larger and heavier, but sufficiently stable and thus sublimable molecules that form solids at RT such as for instance phthalocyanines $[12,13]$ and porphyrins [14] need to be placed in the UHV system, and their evaporation is initiated and controlled by heating the source material, e.g. in a Knudsen cell. Thermal decomposition in the crucible or fragmentation upon adsorption on the substrate [15] significantly limits the classes of molecules that can be sublimed in this way. More advanced deposition techniques like pulse injection $[16,17]$, electrospray deposition (ESD) $[18,19]$, and electrospray ion beam deposition (ES-IBD) $[20,21]$ enable the transfer of large molecules like DNA [17] and proteins [21] or metal clusters [20] directly from solution onto surfaces in a vacuum environment. For pulse injection and ESD the solvent reaches the location of the sample and causes poor vacuum conditions during the deposition (typically $>10^{-7}$ mbar), thereby imposing limitations concerning the reactivity of the substrates against the solvent [21]. Pulse injection and ESD are therefore mainly used in combination with inert surfaces such as $\mathrm{Au}(111)$, graphite, oxidized $\mathrm{Si}$, or $\mathrm{KBr}$. The mass selection stage employed in ES-IBD allows for an effective separation of the target molecules from the solvent molecules, leading to a significant improvement of the vacuum conditions to $10^{-10}$ mbar during deposition [21]. However, the experimental effort for ES-IBD is complex and expensive. The here presented deposition procedure is based on comparatively simple and inexpensive instrumentation and is suitable for the UHVcompatible and contamination-free deposition of organic molecules, which form at RT a solid with a high vapor pressure, onto highly reactive transition-metal surfaces.

Specifically, we report on the adsorption of pyrene $\left(\mathrm{C}_{16} \mathrm{H}_{10}\right.$, see Figure $4 \mathrm{c}$ for the chemical structure) on metal surfaces, since strong hybridization effects with ferromagnetic transition-metal surfaces are expected for this planar polycyclic aromatic hydrocarbon consisting of four fused benzene rings that share an extended $\pi$-system. In addition, pyrene can be considered as a chemical precursor for pyrene-based double-decker molecules that could act as singe-molecule spin-filter elements [22]. Pyrene is at RT a colorless solid with a high vapor pressure of $6.0 \times$ $10^{-6}$ mbar $\left(>10^{-4}\right.$ mbar for $\left.T>323 \mathrm{~K}\right)[23,24]$. This makes the handling of pyrene under UHV conditions ( $p<$ $10^{-9}$ mbar) challenging, in particular during the system bakeout at $T>400 \mathrm{~K}$ that is required to reach $\mathrm{UHV}$ after air exposure. To the best of our knowledge, there is no previous report on UHV deposition and observation of single pyrene molecules and pyrene adlayers on metal surfaces. Wang et al. [25] have prepared and imaged ordered pyrene adlayers on $\mathrm{Cu}(111)$ in $0.1 \mathrm{M}$ perchloric acid $\left(\mathrm{HClO}_{4}\right)$ solution in an electrochemical cell
- a procedure that is incompatible with the highly reactive surfaces of ultrathin ferromagnetic Fe or Co films. Selfassembly of bromine-substituted pyrene derivatives 2,7dibromopyrene $\left(\mathrm{C}_{16} \mathrm{H}_{8} \mathrm{Br}_{2}\right)$ and 1,3,6,8-tetrabromopyrene and $\left(\mathrm{C}_{16} \mathrm{H}_{6} \mathrm{Br}_{4}\right)$ on $\mathrm{Au}(111)$ was studied by scanning tunneling microscopy (STM) under UHV conditions by Pham et al. [26]. However, these compounds have a 2-3 orders of magnitude lower vapor pressure $\left(1.6 \times 10^{-8}\right.$ and $5.3 \times$ $10^{-9}$ mbar, respectively, at RT [24]) than pyrene and for that reason could be conventionally deposited by thermal evaporation from a heated glass crucible [26]. However, the bromination withdraws electron charge from the $\pi$-system and is expected to reduce the hybridization effects, making the unsubstituted pyrene the more promising choice.

Here, we demonstrate the successful formation of clean pyrene adlayers firstly on the weakly reactive $\mathrm{Au}(111)$ surface and secondly on more reactive $1-4$ monolayers (MLs) thin Fe films on W(110) by low-temperature scanning tunneling (LT-STM) imaging. Fe/W(110) is a wellknown model system for ferromagnetic surfaces [27,28] that exhibits a rich magnetization structure with domains on the hundreds of nanometer length scale and in-plane or out-of-plane magnetization depending on the Fe thickness (1...4ML). Fe/W(110) substrates have previously been used for experimental as well as theoretical studies of hybrid molecular magnets $[6,9,15,29]$.

2 Experimental Methods All experiments are performed in a multi-chamber UHV system featuring facilities for single crystal surface cleaning, thin film deposition, molecule sublimation, and LT-STM. The Au(111) single crystal is cleaned by cycles of $\mathrm{Ar}^{+}$sputtering (20 min., $800 \mathrm{eV}$ energy, $5 \times 10^{-6} \mathrm{~A} \mathrm{~cm}^{-2}$ ion current density, and $10^{-6} \mathrm{mbar}$ Ar pressure) and annealing at $800 \mathrm{~K}$ for $30 \mathrm{~min}$. The cleaning procedure for the W(110) single crystal follows the procedures described in [30] and involves repeated flashing at about $2500 \mathrm{~K}$ for $15 \mathrm{~s}$ and heating in an $10^{-6}$ mbar oxygen atmosphere at about $2000 \mathrm{~K}$ to remove carbon contamination. The ultrathin Fe films are epitaxially grown on W(110) held at RT using an electron beam evaporator. Upon subsequent post-annealing at $570 \mathrm{~K}$ for 7 min., a pseudomorphic first Fe ML is formed, on which additional $\mathrm{Fe}$ atoms coalesce to $\mathrm{Fe}$ islands and agglomerate at the step edges imposed by the W crystal [27]. All STM experiments are performed in-situ at $78 \mathrm{~K}$ using polycrystalline W-tips that are flashed in UHV to remove impurities prior to transferring them into the STM. If the image quality deteriorates during measurement, we apply short voltage pulses to the tip to improve its resolution capability and stability, and subsequently we move the tip to a several $\mu \mathrm{m}$ distant new location that exhibits the unperturbed molecule arrangement. All STM images are recorded in the constantcurrent mode with a set-point current $I_{\mathrm{t}}=1.0 \mathrm{nA}$ and at various bias voltages $V_{\text {bias }}$.

As mentioned above, the deposition of pyrene in UHV is challenging due to the high vapor pressure. Commer- 
cially available pyrene powder (98\% purity, Acros Organics) was further purified by column chromatography over silica using dichloromethane:hexane (1:1) as eluent. Then it was recrystallized several times from hexane before it is brought into UHV. We employ a Knudsen cell located in a dedicated molecule-deposition UHV chamber that can be sealed from the other chambers with a valve in order to reduce pyrene exposure of the entire vacuum system. After thoroughly degassing the Knudsen cell including an empty glass crucible under UHV, we briefly vent only the molecule-deposition chamber to fill the crucible with about $30 \mathrm{mg}$ of pyrene powder. Following a standard procedure to reach UHV conditions, the chamber is then pumped and baked at $400 \mathrm{~K}$ for 30 hours. The bottom-mounted flange of the Knudsen cell is outside the baking zone so that the temperature of the crucible in the Knudsen cell does not exceed $303 \mathrm{~K}$ during the bakeout. In this way, we prevent excessive sublimation of pyrene during the bakeout. Visual inspection of the crucible after bakeout through a vacuum viewport did not reveal any change of the filling level or the appearance of the pyrene powder. The crucible had only to be refilled after 6-8 weeks due to the constant evaporation at RT. After cooling the molecule-deposition chamber to RT we reach a base pressure of $1 \times 10^{-10}$ mbar. The fact that this pressure is much lower than the vapor pressure of pyrene indicates that the turbo molecular pump and the chamber walls with an approximately $10^{5}$ larger area than the crucible opening of $4.5 \mathrm{~mm}^{2}$ act as sinks for pyrene molecules. Therefore, the Knudsen cell can be considered as a source of a molecular beam even at RT, i.e. without heating the crucible. The preparation of pyrene adlayers is achieved by placing a pre-cleaned substrate $15 \mathrm{~cm}$ in front of the Knudsen cell kept at RT, and a shutter is used to control the exposure time to the molecular beam. We estimate from the observed density of adsorbed molecules on the $\mathrm{Fe}$ surfaces a deposition rate of about $0.05 \mathrm{ML} / \mathrm{s}$. During deposition all substrates are at RT. Immediately after closing the shutter, the sample is transferred to the LT-STM chamber with a base pressure of $10^{-11}$ mbar and cooled down to $78 \mathrm{~K}$ for analysis.

3 Experimental Results In this section we describe the results of the deposition studies for pyrene (i) on the inert $\mathrm{Au}(111)$ surface, which is weakly prone to contamination that is possibly caused by our specific deposition procedure, and (ii) on ultrathin Fe films on W(110), which are more reactive and thus more contamination-sensitive, but also represent a well-defined magnetic model substrate for the formation of hybrid molecular magnets.

3.1 Pyrene on Au(111) STM investigations of the surface adsorption of sensitive molecules require clean and atomically flat surfaces. The (111) surface of an Au single crystal is an often used and easy to prepare model system. After several cycles of $\mathrm{Ar}^{+}$sputtering and subsequent annealing the Au(111) surface exhibits large atomic terraces with the characteristic herringbone reconstruction [31-33] as shown in Figure 1a.

After exposing the clean $\mathrm{Au}(111)$ surface for $1 \mathrm{~min}$. to the pyrene-filled crucible held at RT, a clean pyrene adlayer with only few defects is formed, see Figure 1b. Each molecule appears as a featureless roundish, weakly hexagonal protrusion with a corrugation of about $60 \mathrm{pm}$ indicating a flat-lying adsorption orientation on the underlying $\mathrm{Au}(111)$ surface. The molecules form a two-dimensional
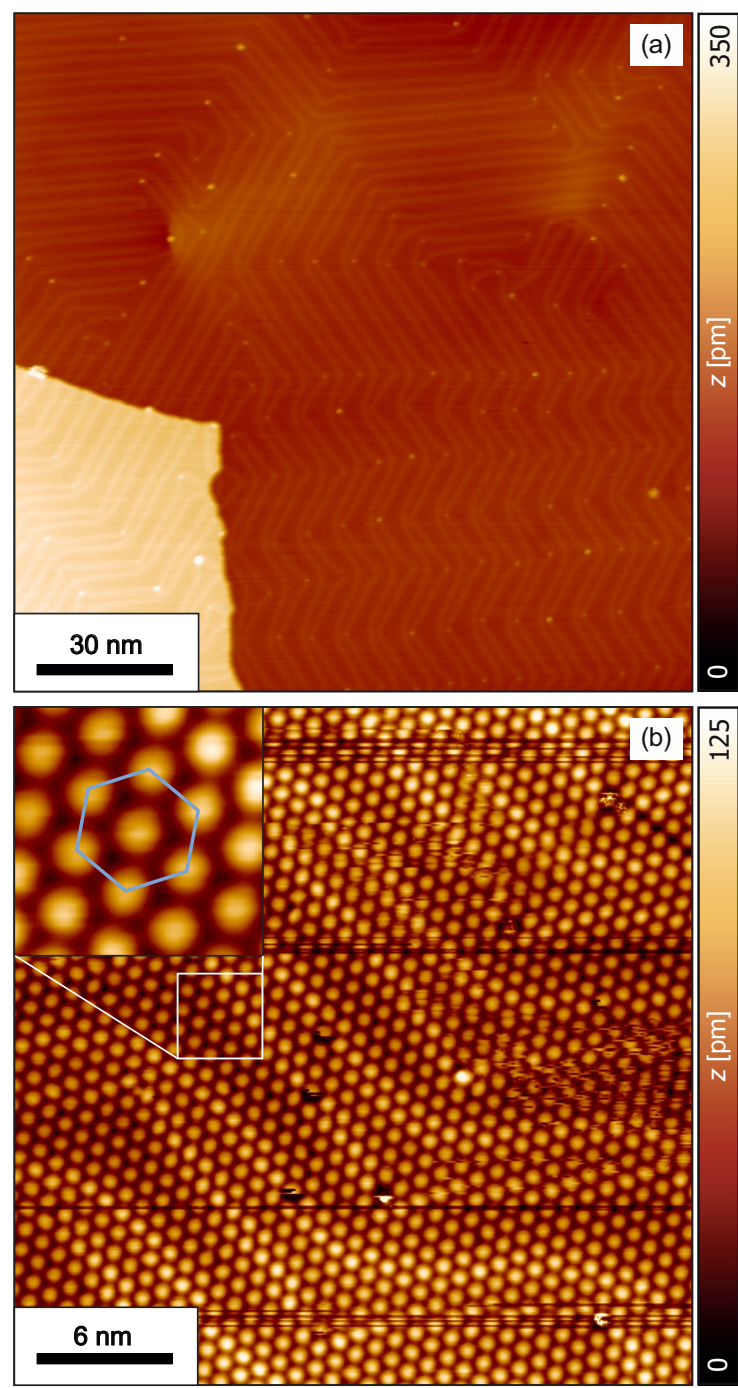

Figure 1 (a) STM image of the Au(111) surface revealing the herringbone reconstruction after several cycles of $\mathrm{Ar}^{+}$sputtering and annealing. The zig-zag structures on the terraces are due to the herringbone reconstruction $\left(150 \times 150 \mathrm{~nm}^{2}\right.$, $V_{\text {bias }}=1.0 \mathrm{~V}, I_{\mathrm{t}}=1.0 \mathrm{nA}$ ). (b) $1 \mathrm{ML}$ of pyrene molecules after $1 \mathrm{~min}$. deposition time on the atomically flat $\mathrm{Au}(111)$ surface shown in (a). Each roundish protrusion represents a pyrene molecule. Inset: Gauss-filtered close-up of the hexagonal superstructure lattice with a lattice constant $a=1050 \mathrm{pm}(30 \times$ $\left.30 \mathrm{~nm}^{2}, V_{\text {bias }}=1.0 \mathrm{~V}, I_{\mathrm{t}}=1.0 \mathrm{nA}\right)$. 
hexagonal superstructure with long-range order extending over hundreds of $\mathrm{nm}$. The lattice constant $a=1050 \mathrm{pm}$ suggests an incommensurate superstructure on $\mathrm{Au}(111)$ (nearest-neighbor distance of $289 \mathrm{pm}$ ), similar to the previously reported case of tetrabromopyrene on $\mathrm{Au}(111)$ [26], which arises due to weak molecule-substrate interaction. The formation of a $1 \mathrm{ML}$ thick adlayer of physisorbed pyrene molecules is further corroborated by the fact that the herringbone reconstruction is visible through the molecular adlayer (faint bright zig-zag lines in the background of Figure 1b, note the different images sizes of Figure 1a and b). If we increase the deposition time well beyond $1 \mathrm{~min}$., the appearance of the adlayer does not change and the herringbone reconstruction remains visible. We conclude that the pyrene coverage does not increase beyond $1 \mathrm{ML}$. We explain this self-limitation of the growth to the $1 \mathrm{ML}$ thick adlayer shown in Figure $1 \mathrm{~b}$ by the immediate desorption of pyrene molecules that arrive on top of the first ML. The weak pyrene-pyrene interaction that inhibits the growth of a second layer at RT is also reflected by the high vapor pressure of pyrene. The horizontal lines and the streaky noise in Figure $1 \mathrm{~b}$ most likely occur when the tip picks up or moves pyrene molecules. The fact that we observe such perturbations more frequently on Au than on Fe substrates corroborates the weaker molecule-surface interaction on the $\mathrm{Au}(111)$ surface, i.e. physisorption of pyrene on $\mathrm{Au}(111)$.

3.2 Fe ultrathin films on $\mathbf{W}(\mathbf{1 1 0})$ The growth mode of ultrathin Fe films on W(110) is governed by the large lattice mismatch between $\mathrm{Fe}$ and $\mathrm{W}\left(a_{\mathrm{W}}-a_{\mathrm{Fe}}\right) / a_{\mathrm{W}}=9.4 \%$ and the different surface free energies $\left(\gamma_{\mathrm{Fe}}=2.0 \mathrm{Jm}^{-2}\right.$

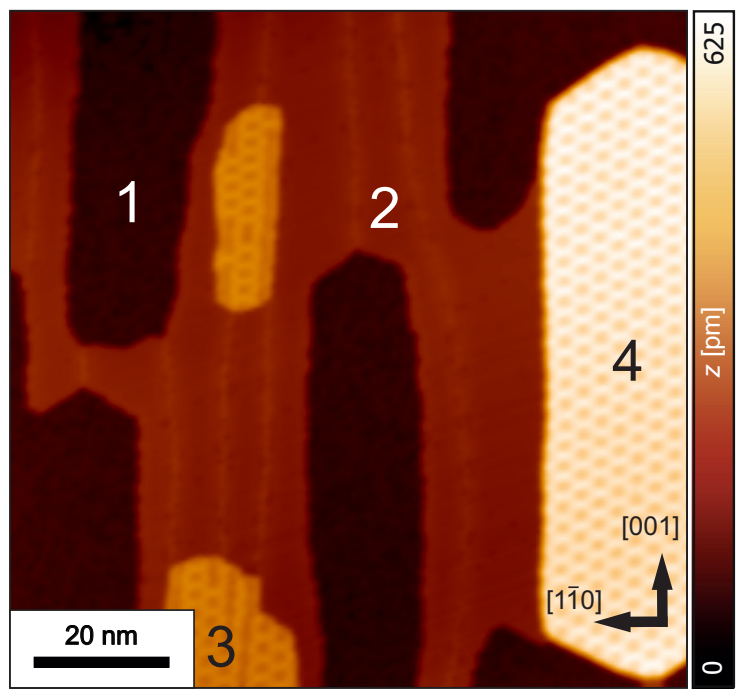

Figure 2 STM image of an epitaxial Fe film on W(110). Numbers indicate the local Fe film thickness in ML. Each thickness reveals a different surface structure that results from different densities and arrangements of the stain-induced dislocations. $\left(100 \times 100 \mathrm{~nm}^{2}, V_{\text {bias }}=100 \mathrm{mV}, I_{\mathrm{t}}=1.0 \mathrm{nA}\right)$. and $\gamma_{\mathrm{W}}=2.9 \mathrm{Jm}^{-2}$ ). The interplay between the resulting atomic and magnetization structures has been studied in great detail $[27,28]$. We briefly summarize the main properties for Fe thicknesses between 1 and $4 \mathrm{ML}$ shown in the overview STM image of an epitaxially grown Fe film in Figure 2. Monatomic steps separate atomically flat terraces of different local Fe thickness (indicated in ML by the superimposed numbers). The first ML grows pseudomorphically with respect to the W(110) substrate and isotropic [34] as reflected by the homogeneous dark brown color of terrace labelled "1". The second ML Fe starts growing only after the first ML is completed. Stress resulting from the lattice mismatch is released by including additional rows of atoms in the second ML leading to anisotropic growth (elongated islands) and characteristic dislocation lines running mainly along the [001] direction (faint bright lines on terrace labelled "2"), which allow an easy identification of areas with an Fe thickness of $2 \mathrm{ML}$ $[27,35]$. All layers above the second ML start growing before the previous layer is completed. In the third ML additional dislocation lines are introduced (terrace labelled "3") [27]. The growth is still anisotropic in [001] direction. Starting from the fourth ML, the insertion of an $\mathrm{Fe}$ atom in roughly every ninth row leads to a two-dimensional distortion network [27] (regular pattern on terrace labelled "4"), which allows the Fe to fully relax. Accordingly, the growth becomes isotropic again [27], see Figure S1 in the Supporting Information.

3.2.1 Pyrene on 1 ML Fe on W(110) Figure 3 depicts adsorbed pyrene molecules on the first ML (second $\mathrm{ML}$ in the top left corner) Fe on W(110). On the first

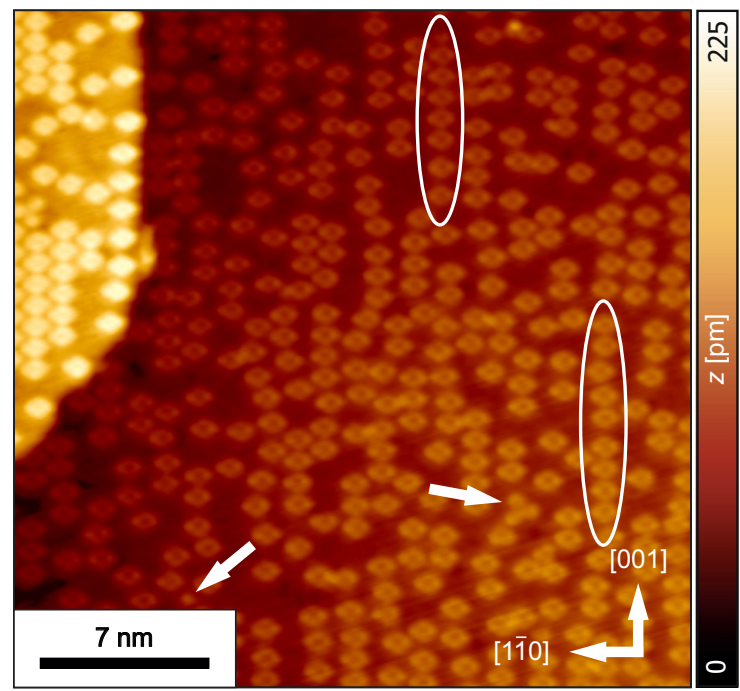

Figure 3 STM image of irregularly adsorbed pyrene molecules after $10 \mathrm{~s}$ deposition on $1 \mathrm{ML} \mathrm{Fe}$ on W(110). Arrows indicate some of the few molecular fragments or impurities and ellipses highlight the short-range ordering along the [001] direction $(28 \times$ $\left.28 \mathrm{~nm}^{2}, V_{\text {bias }}=-300 \mathrm{mV}, I_{\mathrm{t}}=1.0 \mathrm{nA}\right)$. 
Fe ML, the pyrene molecules adsorb in a unique and flat configuration as indicated by the similar appearance of all molecules and the small corrugation of $60 \mathrm{pm}$. Almost all molecules are intact, and contamination can hardly be discerned. Arrows point at some molecular fragments or impurities. The lateral arrangement of the molecules is rather irregular most likely due to the pseudomorphic structure of the first Fe ML below. However, there is a preference to form ordered lines along the [001] direction (ellipses in Figure 3). The molecules show a rather uniform in-plane rotational orientation with the long axis aligned roughly

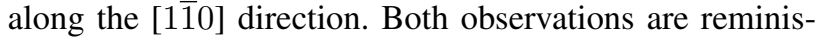
cent of the 2-fold symmetry of the W(110) substrate.

3.2.2 Pyrene on 2 ML Fe on W(110) The growth of pyrene on 2 ML Fe on W(110) the sub-ML regime results in a particular packing arrangement, see Figure 4. Isolated pyrene molecules are irregularly arranged on top and near the dislocation lines (almost vertical brighter stripes in Figure $4 \mathrm{a}$ ), whereas patches of self-assembled molecules are formed in the unperturbed areas between the dislocation lines. Images at lower pyrene coverage (see Figure S2 in the Supporting Information) reveal that the areas of the dislocation lines are first occupied and saturated by molecules before the ordered molecular arrays are formed in between. This behavior is similar to the observation of Heß et al. [9] that 2,4,6-triphenyl-1,3,5-triazine molecules on $2 \mathrm{ML} \mathrm{Fe}$ on W(110) prefer to adsorb near the dislocation lines due to the locally higher coordination number of the substrate. All ordered patches consist of molecular rows running in [001] direction, i.e. parallel to the dislocation lines, forming the same adsorption lattice. A close-up of this superstructure in Figure $4 \mathrm{~b}$ reveals a rhombic unit cell with $a=862 \mathrm{pm}$ and $b=1184 \mathrm{pm}$ in agreement with the 2-fold symmetry of the $\mathrm{Fe}(110)$ surface lattice. The lattice vector a points in [001] direction indicating that the molecular overlayer is commensurate to the $\mathrm{Fe}(110)$ atomic lattice. The molecular corrugation is $60 \mathrm{pm}$. Each pyrene molecule appears rhombic; a shape that can directly be related to the chemical structure of pyrene shown in Figure 4c. The apparent depression in the center of the molecule of about $10 \mathrm{pm}$ (see Figure S3 in the Supporting Information) indicates larger molecular orbitals with enhanced charge density or higher adsorption height of the $\mathrm{H}$-coordinated $\mathrm{C}$ atoms compared to the 6 central $\mathrm{C}$ atoms that are shared among two or three $\mathrm{C}$ rings. All molecules have the same in-plane adsorption orientation with the short axes of the rhombus parallel to the [001] direction and the long axes offset from the superstructure lattice vectors b by approximately $20^{\circ}$. A possible model of a commensurate pyrene adlayer on $2 \mathrm{ML} \mathrm{Fe}$ on $\mathrm{W}(110)$ that reproduces all our observations is shown in Figure 4d. Note that the adsorption site (C rings centered above Fe bridge sites) could not be determined from the experiments, but is chosen according to the reported adsorption site of benzene on $2 \mathrm{ML} \mathrm{Fe}$ on W(110) [6].

3.2.3 Pyrene on 3 and 4 ML Fe on W(110) In Figure 5, we present an overview image of a sub-ML pyrene
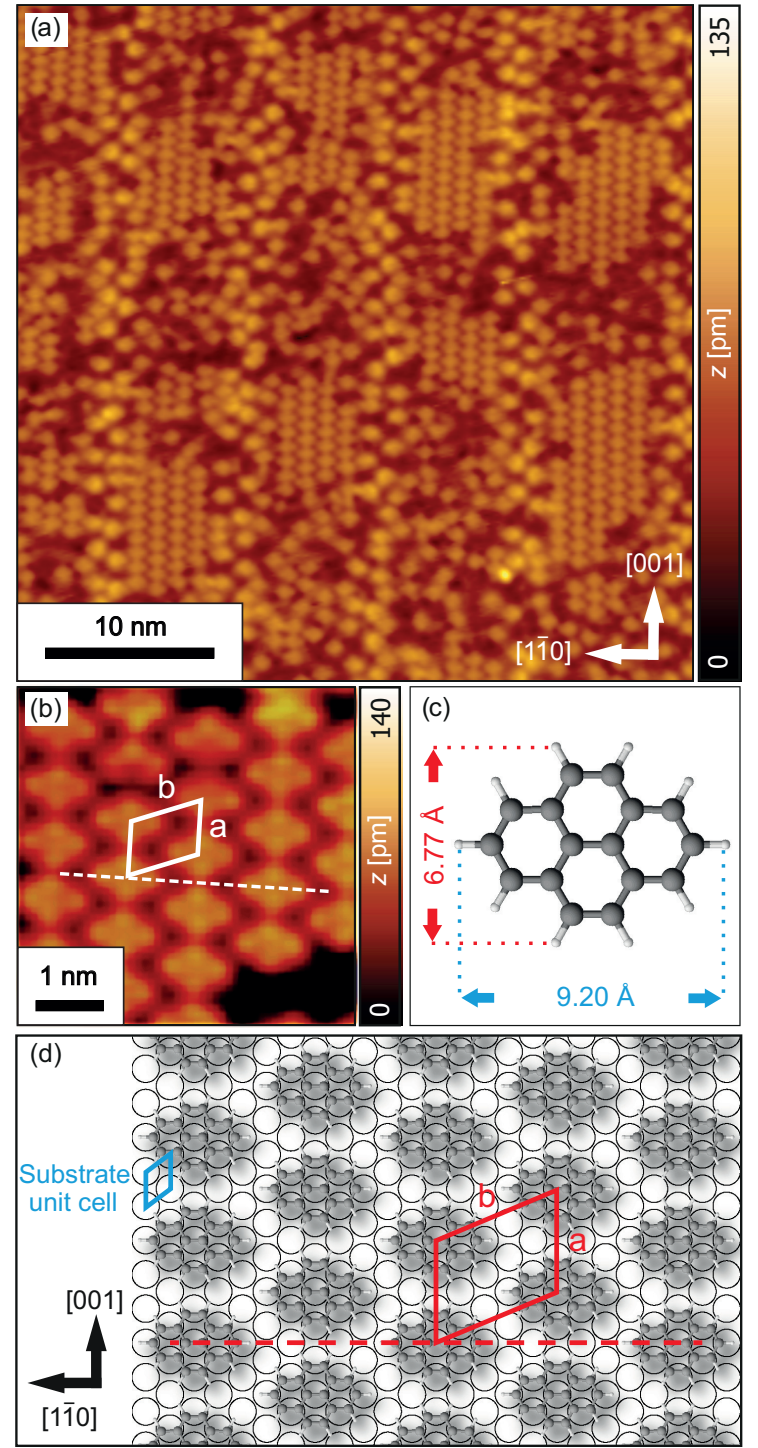

Figure 4 (a) Overview STM image of a sub-ML pyrene molecules after $10 \mathrm{~s}$ deposition on $2 \mathrm{ML}$ Fe on W(110) showing irregular adsorption near the dislocation lines and self-assembled patches of molecules in between $\left(40 \times 40 \mathrm{~nm}^{2}, V_{\text {bias }}=100 \mathrm{mV}\right.$, $I_{\mathrm{t}}=1.0 \mathrm{nA}$ ). (b) Close-up of pyrene molecules on $2 \mathrm{ML}$ Fe on $\mathrm{W}(110)$. The rhombic unit cell of the superstructure is indicated. The dashed line is the direction of the long axis of the molecules $\left(5.0 \times 5.0 \mathrm{~nm}^{2}, V_{\text {bias }}=-100 \mathrm{mV}, I_{\mathrm{t}}=1.0 \mathrm{nA}\right)$. (c) Chemical structure (grey: carbon, white: hydrogen) and physical dimensions (H-to-H distances) of pyrene [36]. (d) Model for the self-assembled and commensurate pyrene superstructure with the molecular adlayer unit cell marked in red and the Fe(110) surface unit cell $\left(287 \times 203 \mathrm{pm}^{2}\right)$ in blue.

coverage on a stepped Fe film on W(110) with the local Fe thickness ranging from 1 to $3 \mathrm{ML}$ and in the inset an image taken on the fourth ML of Fe. Obviously, the adsorption pattern changes again on the third and fourth $\mathrm{ML}$ 


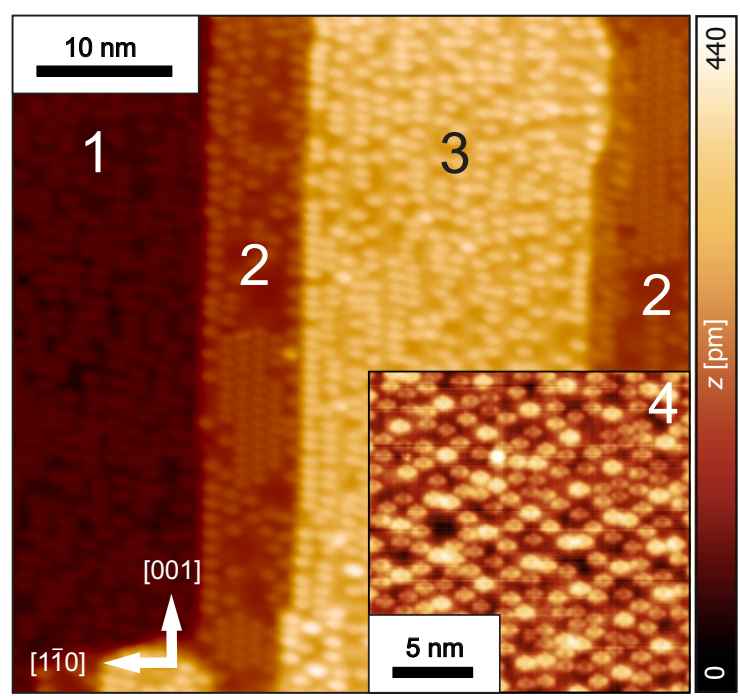

Figure 5 Adsorption pattern of a sub-ML pyrene molecules after $10 \mathrm{~s}$ deposition on an ultrathin Fe film on W(110). Numbers indicate the local Fe thickness in ML. Each thickness reveals a different adsorption pattern due to the different densities and arrangements of strain-induced dislocations of the Fe film $\left(50 \times 50 \mathrm{~nm}^{2}\right.$, $\left.V_{\text {bias }}=100 \mathrm{mV}, I_{\mathrm{t}}=1.0 \mathrm{nA}\right)$. Inset: Irregular desorption pattern on $4 \mathrm{ML}$ Fe on $\mathrm{W}(110)\left(20 \times 20 \mathrm{~nm}^{2}, V_{\text {bias }}=100 \mathrm{mV}\right.$, $I_{\mathrm{t}}=1.0 \mathrm{nA}, z$-range $\left.=200 \mathrm{pm}\right)$.

of Fe. On 3 ML Fe, a mixture of small self-assembled ordered patches, regular rows along the step edges, and irregular adsorption is visible that originates from the irregular dislocation lines in the underlying $\mathrm{Fe}$, which are denser than for $2 \mathrm{ML} \mathrm{Fe}$ on W(110), see Figure 2. For pyrene on $4 \mathrm{ML} \mathrm{Fe}$, the adsorption pattern is laterally irregular, but the different apparent height (brightness) of the molecules weakly reflects the hexagonal distortion network of $4 \mathrm{ML}$ $\mathrm{Fe}$ on W(110), see Figure 2. A closer look at the pyrene coverage indicates an increase on 3 and in particular $4 \mathrm{ML}$ Fe compared to 1 and $2 \mathrm{ML}$ Fe. This can be explained by the increasing density of dislocations from 2 to $4 \mathrm{ML}$ $\mathrm{Fe}$, which are know to provide preferred, i.e. energetically more stable adsorption sites [9]. Finally, we note that the rhombic shape of the adsorbed molecule, its orientation with respect to the principal axes of the substrate, and the apparent depression in the center are common features observed for pyrene adsorbed on all Fe layer thicknesses from 1 to $4 \mathrm{ML}$.

4 Conclusion We have presented a UHV-compatible sublimation procedure for pyrene molecules at RT that allows the controlled deposition in the sub-ML regime in spite of the unfavorably high vapor pressure of pyrene. In-situ STM images of pyrene adlayers on inert Au(111) and also on more reactive ultrathin, epitaxial Fe films on $\mathrm{W}(110)$ confirm the cleanness of the deposition procedure. There are only very few contamination-induced perturbations with a similar low density than found for molecular adlayers deposited by conventional sublimation at elevated temperature. On both $\mathrm{Au}(111)$ and $\mathrm{Fe} / \mathrm{W}(110)$ substrate systems pyrene adsorbs in a flat and intact configuration.

On the $\mathrm{Au}(111)$ surface, the interaction between pyrene and the substrate is rather weak as indicated by the fact that the herringbone reconstruction of the $\mathrm{Au}(111)$ surface is not lifted upon molecule adsorption. The physisorbed molecules self-assemble in large (several hundreds of $\mathrm{nm}^{2}$ ), hexagonally ordered, but incommensurate arrays. Weak pyrene-pyrene interaction causes the second-layer pyrene molecules to desorb at RT, thereby self-limiting the pyrene growth on $\mathrm{Au}(111)$ to the first ML.

On the Fe ultrathin film substrates, stronger moleculesubstrate interaction is expected to give rise to pyrene chemisorption. On the basis of previous experimental results $[6,8,9,15,29]$ and DFT calculations $[5,7,10,22]$ we assume that chemisorption occurs through interactions between $\left(d_{\pi}=d_{\mathrm{xz}}+d_{\mathrm{yz}}+d_{\mathrm{z}^{2}}\right)$ orbitals of the Fe surface with the $\pi$-conjugated molecular orbitals of the pyrene. Formation of covalent bonds between molecule and substrate would severely modify the molecular geometry and symmetry [37], which is not observed in the presented STM images. The growth of pyrene on Fe ultrathin film substrates is strongly affected by the structure of the $\mathrm{Fe}$ substrate as demonstrated by the clear dependence of the molecular adsorption patterns on the Fe layer thickness. Irregular adsorption is found on the pseudomorphic first Fe ML as well as on the dislocations and their range of influence in the second and following Fe MLs. The density of dislocations increases with the Fe thickness. On the second Fe ML the unperturbed areas between the dislocation lines allow the formation of larger patches of self-assembled molecules. The size of the patches is limited by the neighboring dislocation lines. The commensurate, rhombic unit cell of the molecular superstructure reflects the symmetry of the $\mathrm{Fe}(110)$ lattice and, hence, corroborates chemisorption. The equal appearance of all molecules in the ordered patches indicates a well-defined adsorption geometry of pyrene on $2 \mathrm{ML}$ Fe on W(110). These adsorption properties of pyrene together with the well-known and well-defined magnetic domain structure with out-of-plane magnetization render the second Fe ML on W(111) ideal for upcoming spin-polarized STM investigations and corresponding DFT calculations on the formation of hybrid molecular magnets and on spin-filter effects in pyrene-based double-decker molecules.

Acknowledgements Financial support from the Volkswagen Foundation through the project "Optically Controlled Spin Logic" is gratefully acknowledged. B.B. acknowledges funding through the Core Program PN18-11 of the Romanian Ministry of Research and Innovation.

\section{References}

[1] M. Petty, M. Bryce, and D. Bloor, Introduction to Molecular Electronics (Oxford University Press, New York, 1995). 
[2] C. Joachim, J. K. Gimzewski, and A. Aviram, Nature 408, 541-548 (2000).

[3] A. R. Rocha, V. M. García-Suárez, S. W. Bailey, C. J. Lambert, J. Ferrer, and S. Sanvito, Nature Mater. 4, 335-339 (2005).

[4] L. Bogani and W. Wernsdorfer, Nature Mater. 7, 179 (2008).

[5] M. Callsen, V. Caciuc, N. Kiselev, N. Atodiresei, and S. Blügel, Phys. Rev. Lett. 111, 106805 (2013).

[6] N. Atodiresei, J. Brede, P. Lazić, V. Caciuc, G. Hoffmann, R. Wiesendanger, and S. Blügel, Phys. Rev. Lett. 105, 066601 (2010).

[7] N. Atodiresei, V. Caciuc, P. Lazić, and S. Blügel, Phys. Rev. B 84, 172402 (2011).

[8] J. Brede, N. Atodiresei, V. Caciuc, M. Bazarnik, A. AlZubi, S. Blügel, and R. Wiesendanger, Nature Nanotech. 9, 1018 (2014).

[9] V. Heß, R. Friedrich, F. Matthes, V. Caciuc, N. Atodiresei, D. E. Bürgler, S. Blügel, and C. M. Schneider, New J. Phys. 19, 053016 (2017).

[10] R. Friedrich, V. Caciuc, N. S. Kiselev, N. Atodiresei, and S. Blügel, Phys. Rev. B 91, 115432 (2015).

[11] M. Doering, H. P. Rust, B. G. Briner, and A. M. Bradshaw, Surf. Sci. 410, L736-L740 (1998).

[12] C. Iacovita, M. V. Rastei, B. W. Heinrich, T. Brumme, J. Kortus, L. Limot, and J. P. Bucher, Phys. Rev. Lett. 101, 116602 (2008).

[13] S. Fahrendorf, N. Atodiresei, C. Besson, V. Caciuc, F. Matthes, S. Blügel, P. Kögerler, D. E. Bürgler, and C. M. Schneider, Nature Commun. 4, 2425 (2013).

[14] A. Scheybal, T. Ramsvik, R. Bertschinger, M. Putero, F. Nolting, and T. A. Jung, Chem. Phys. Lett. 411, 214-220 (2005).

[15] S. Fahrendorf, F. Matthes, D. E. Bürgler, C. M. Schneider, N. Atodiresei, V. Caciuc, S. Blügel, C. Besson, and P. Kögerler, SPIN 4, 1440007 (2014).

[16] H. Tanaka and T. Kawai, J. Vac. Sci. Technol. B 15, 602 (1997).

[17] H. Tanaka and T. Kawai, Nature Nanotech. 4, 518 (2009).

[18] J. N. O'Shea, J. B. Taylor, J. C. Swarbrick, G. Magnano, L. C. Mayor, and K. Schulte, Nanotechnology 18, 035707 (2007).

[19] A. Hinaut, T. Meier, R. Pawlak, S. Feund, R. Johr, S. Kawai, T. Glatzel, S. Decurtins, K. Mullen, A. Narita, S. X. Liu, and E. Meyer, Nanoscale 10, 1337 (2018).

[20] S. Rauschenbach, F. L. Stadler, E. Lunedei, N. Malinowski, S. Koltsov, G. Costantini, and K. Kern, Small 2, 540-547 (2006).

[21] S. Rauschenbach, M. Ternes, L. Harnau, and K. Kern, Annual Rev. Anal. Chem. 9, 473-498 (2016).

[22] K. V. Raman, A. M. Kamerbeek, A. Mukherjee, N. Atodiresei, T. K. Sen, P. Lazić, V. Caciuc, R. Michel, D. Stalke, S. K. Mandal, S. Blügel, M. Münzenberg, and J. S. Moodera, Nature 493, 509 (2013).

[23] W. J. Sonnefeld, W. H. Zoller, and W. E. May, Anal. Chem. 55, 275-280 (1983).

[24] U.S. Environmental Protection Agency. Chemistry Dashboard. https://comptox.epa.gov.

[25] D. Wang, L. J. Wan, Q. M. Xu, C. Wang, and C.L. Bai, Surf. Sci. 478, L320-L326 (2001).
[26] T. A. Pham, F. Song, M. T. Nguyen, and M. Stöhr, Chem. Commun. 50, 14089-14092 (2014).

[27] H. Bethge, D. Heuer, C. Jensen, K. Reshöft, and U. Köhler, Surf. Sci. 331, 878-884 (1995).

[28] O. Pietzsch, A. Kubetzka, M. Bode, and R. Wiesendanger, Phys. Rev. Lett. 84, 5212-5215 (2000).

[29] J. Brede, N. Atodiresei, S. Kuck, P. Lazić, V. Caciuc, Y. Morikawa, G. Hoffmann, S. Blügel, and R. Wiesendanger, Phys. Rev. Lett. 105, 047204 (2010).

[30] M. Bode, S. Krause, L. Berbil-Bautista, S. Heinze, and R. Wiesendanger, Surf. Sci. 601, 3308-3314 (2007).

[31] M. A. Van Hove, R. J. Koestner, P. C. Stair, J. P. Bibérian, L. L. Kesmodel, I. Bartoŝ, and G. A. Somorjai, Surf. Sci. 103, 189-217 (1981).

[32] W. Kaiser and R. Jaklevic, Surf. Sci. Lett. 182, L227-L233 (1987).

[33] F. Reinert and G. Nicolay, Appl. Phys. A 78, 817-821 (2004).

[34] U. Gradmann and G. Waller, Surf. Sci. 116, 539-548 (1982).

[35] H. L. Meyerheim, D. Sander, R. Popescu, J. Kirschner, P. Steadman, and S. Ferrer, Phys. Rev. B 64, 045414 (2001).

[36] National Center for Biotechnology Information. PubChem Compound Database; CID $=31423$, https://pubchem.ncbi.nlm.nih.gov/compound/31423.

[37] A. Zhao, Q. Li, L. Chen, H. Xiang, W. Wang, S. Pan, B. Wang, X. Xiao, J. Yang, J. G. Hou, and Q. Zhu, Science 309, 1542 (2005) 


\section{Graphical Table of Contents}

GTOC image:

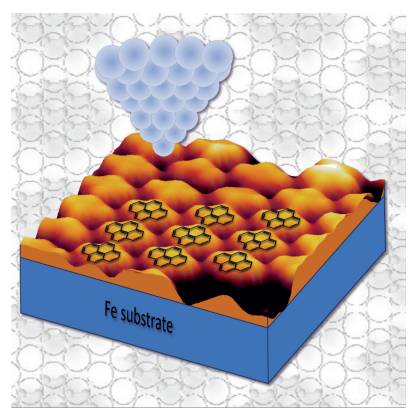

Pyrene on Fe surfaces is an interesting model system to study spin-dependent hybridization effects relevant for molecular spintronics. However, the high vapor pressure complicates pyrene deposition in ultra-high vacuum (UHV) required for reactive metal surfaces. Here, an UHV sublimation procedure is presented and resulting pyrene adlayers on $\mathrm{Au}(111)$ and $\mathrm{Fe} / \mathrm{W}(110)$ substrates are characterized by scanning tunneling microscopy. 